\title{
Innovationen in der Freien Wohlfahrtspflege
}

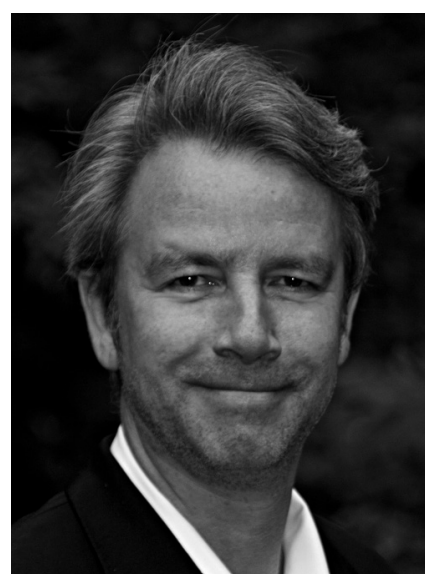

VON GERHARD TIMM

Dr. Gerhard Timm ist

Geschäftsführer der Bundesarbeitsgemeinschaft der Freien Wohlfahrtspflege e. V. www.bagfw.de

\author{
Etablierte Verbände und Organisationen sehen \\ sich öfter mit der Forderung nach "Innovation" \\ konfrontiert. Übersehen wird dabei, dass \\ gerade in der Freien Wohlfahrtspflege mit \\ ihren verschiedenen Verbänden und regionalen \\ Gliederungen immer wieder gangbare Wege nach \\ besseren Hilfen für Menschen in Not gesucht, \\ gefunden und für andere geebnet wurden.
}

Die Freie Wohlfahrtspflege wirkt auf eine solidarische, gerechte, inklusive Gesellschaft hin. Sie ist in diesem Sinne sozialpolitischer Anwalt und unterstützt Menschen in schwierigen Lebenslagen durch vielfältige Angebote. Dieser Aufgabe kommt sie in komplexen verbandlichen und unternehmerischen Strukturen nach.

Seit ihrer Entstehung haben die Spitzenverbände der Freien Wohlfahrtspflege für ungezählte »Innovateure « die strukturellen Voraussetzungen geschaffen, um neue Ideen zur Lösung eines gesellschaftlichen Problems zu entwi- einer sozialen Herausforderung im Interesse der betroffenen Menschen.

Die Spitzenverbände legen großen Wert auf eine breite Partizipation aller Beteiligten bei der Weiterentwicklung ihrer Angebote. Sie nutzen gezielt das Engagement der vielen haupt- und ehrenamtlichen Mitarbeitenden und deren Nähe zu den hilfesuchenden Menschen, die als Nutzerinnen und Nutzer ihre Einrichtungen und Dienste in Anspruch nehmen. Gerade die Betroffenen selbst, die auf die sozialen Dienstleistungen zurückgreifen, geben beispielsweise im

\section{"Die Freie Wohlfahrtspflege bietet mit ihren stabilen Strukturen eine gute Basis für praktikable Innovationen"}

ckeln und umzusetzen. Das derzeitige Dienstleistungsspektrum ist damit das Ergebnis vielfältigster Innovationen, die aus der praktischen Arbeit heraus entwickelt wurden und über Jahrzehnte in sie eingeflossen sind. Die Freie Wohlfahrtspflege blickt daher auf eine lange Tradition sozialer Innovationen zurück.

Die entscheidende Frage zur Beurteilung der Qualität einer sozialen Innovation ist die Frage nach ihrer Wirkung im Hinblick auf die bessere Bewältigung
Rahmen von Klientenbefragungen oder Beiräten wertvolle Impulse für Innovationen.

Qualifizierung, finanzielle Förderung, Fachberatung sowie die Ermöglichung von Austausch und Diskussion sind Innovationsaktivitäten, die systematisch und nachhaltig in die Arbeitsprozesse eingebunden sind.

Die dezentralen Strukturen der Spitzenverbände sind für eine gewinnbringende Vernetzung lokaler und regionaler Projekte hilfreich. Gleichzeitig können 
gute Ideen mithilfe der spezifischen Strukturen bundesweit übertragen werden.

\section{Instrumente zur Förderung von Innovation}

Das gemeinsame Ziel der Freien Wohlfahrtspflege ist es, die Breite und die Vielfalt sozialer Innovationen in ihren Reihen insgesamt weiter zu fördern und dies stärker als bisher zu systematisieren. Damit Organisationen innovationsfreundlich sind, müssen sie Freiräume schaffen, konflikt- und netzwerkfähig sein und eine gewisse Bereitschaft zum Risiko haben. Dies muss auf allen Ebenen gepflegt werden.

Die Spitzenverbände arbeiten untereinander und mit anderen Organisationen an diesem Thema eng zusammen. Mögliche Kooperationspartner sind neben den politischen Akteuren weitere Sozialverbände, Verbraucherund Selbsthilfeorganisationen, aber auch Fachverbände und Unternehmen der Sozialwirtschaft oder geeignete (Sozial-) Unternehmen. Denn die Freie Wohlfahrtspflege kann durch ihr Know-how und ihre umfassende Infrastruktur nicht nur eigene, sondern auch die Ideen externer Sozialunternehmen unterstützen und zu ihrer Verbreitung beitragen.

\section{Notwendige Rahmenbedingungen für Innovationen}

Für die nachhaltige Förderung sozialer Innovationen müssen über die verbandsinternen Veränderungen hinaus auch die entsprechenden externen Rahmenbedingungen verbessert werden. Reformbedarf sieht die Freie Wohlfahrts- pflege im Zuwendungs-, Beihilfen- und Steuerrecht, die in ihrer derzeitigen Form teilweise innovationshemmend wirken.

Die Freie Wohlfahrtspflege setzt sich für einen Wettbewerb ein, der maßgeblich über die Qualität der angebotenen Leistungen - und nicht nur über den Preis - ausgetragen wird, da ein reiner Preiswettbewerb innovationsschädigend ist. Innovationsförderlich ist demgegenüber ein offen gestaltetes sozialrechtliches Dreiecksverhältnis zwischen Leistungsträgern, Leistungserbringern und Nutzern, das den Nutzern ihr Wunschund Wahlrechte sichert, eine flexible Leistungserbringung seitens der Träger ermöglicht und damit Spielräume für Innovation schafft.

\section{Freie Wohlfahrtspflege: „Unsere Forderungen für bessere Innovationen»}

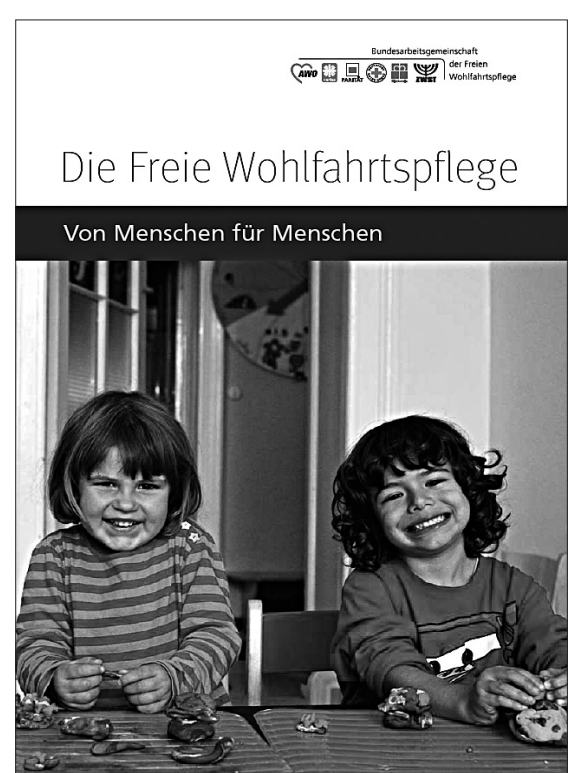

Die Bundesarbeitsgemeinschaft der Freien Wohlfahrtspflege e. V. (BAGFW) ist die gemeinsame Arbeitsplattform der sechs Spitzenverbände der Freien Wohlfahrtspflege: Arbeiterwohlfahrt, Deutscher Caritasverband, Paritätischer Wohlfahrtsverband, Deutsches Rotes Kreuz, Diakonie Deutschland, Zentralwohlfahrtsstelle der Juden in Deutschland. Diese Spitzenverbände vertreten bundesweit über 90.000 soziale Einrichtungen und Dienste mit über 1,4 Millionen hauptberuflich Mitarbeitenden und 2,5 bis 3 Millionen ehrenamtlich Tätigen.

Die Bundesarbeitsgemeinschaft der Freien Wohlfahrtspflege hat im Dezember 2012 in einem Positionspapier ihre Haltung zu dem Thema "Soziale Innovationen in der Freien Wohlfahrtspflege" dargestellt, aus dem die nachfolgenden Schlussfolgerungen entnommen sind:

"Innovation in der sozialen Arbeit ist ein kontinuierlicher Prozess, um der Herausforderung der sich ständig verändernden Lebensbedingungen von Menschen gerecht zu werden. Die Freie Wohlfahrtspflege mit ihrer langjährigen Erfahrung als sozialer Innovateur und ihrem flächendeckenden Netz sozialer Angebote bringt ihr Knowhow gerne in einen bundesweiten Diskurs zur Verbesserung der Rahmenbedingungen für soziale Innovationen ein. Gleiches gilt für entsprechende Bestrebungen auf europäischer Ebene.

Bereits jetzt lassen sich - ohne Anspruch auf Vollständigkeit - folgende Anforderungen an förderliche Rahmenbedingungen für soziale Innovationen zusammenfassen:

- Eine wie von der EU-Kommission vorgeschlagene weitgefasste Beschreibung der Begriffe "soziale Innovationen" und "soziales Unternehmertum", die der Vielfalt an Formen und Akteuren gerecht wird;

- ein transparenter Diskussionsprozess auf nationaler und europäischer Ebene zur Verbesserung der Rahmenbedingungen für soziale Innovationen unter Beteiligung erfahrener Dienstleister des Dritten Sektors;

- die Einrichtung einer ständigen Arbeitsgruppe besetzt aus Vertreterinnen und Vertretern des BMFSFJ, der Freien Wohlfahrtspflege sowie weiterer Akteure, um gemeinsam Kriterien für eine nachhaltige Nutzung von erfolgreichen Innovationen zu generieren und diese in den Politikprozess einzubringen;

- eine gesamtwirtschaftliche und -gesellschaftliche Betrachtung und Bewertung sozialer Innovationen;

- verbesserte nationale Rahmenbedingungen der Förderung, d. h. größere Akzeptanz gegenüber gewissen Risiken, da das Ergebnis innovativer Projektvorhaben zwangsläufig offen ist, bessere Bedingungen für die Überführung innovativer Verfahren und Angebote aus der Projektfinanzierung in die Regelfinanzierung;

n passgenaue europäische Förderinstrumente;

- bessere Anerkennung eines offen gestalteten sozialrechtlichen Dreiecksverhältnisses auf europäischer Ebene, das dem Nutzer Wahlrechte sichert, eine flexible Leistungserbringung seitens der Träger ermöglicht und damit Spielräume für Innovation schafft;

- eine verstärkte Investition in die Wirkungsorientierung und die Stärkung des Bewusstseins für dieses Thema."

Die achtseitige Stellungnahme findet sich auf der Website der Organisation in der Rubrik «Veröffentlichungen und Stellungnahmen«:

www.bagfw.de/uploads/tx twpublication/ 2012-11-30_Positionspapier_der_BAGFw_zu_ sozialen Innovationen Final.p $\bar{d} f$ 